\title{
TELEREHABILITATION CLINICAL AND VOCATIONAL APPLICATIONS FOR ASSISTIVE TECHNOLOGY: RESEARCH, OPPORTUNITIES, AND CHALLENGES
}

\author{
MARK R. SCHMELER ${ }^{1}$, RICHARD M. SCHEIN ${ }^{1}$, MICHAEL MCCUE ${ }^{1}$ \\ \& KENDRA BETZ ${ }^{2}$ \\ ${ }^{1}$ DEPARTMENT OF REHABILITATION SCIENCE AND TECHNOLOGY, UNIVERSITY OF PITTSBURGH \\ ${ }^{2}$ ProstheTICS AND SENSORY AIDS SERVICE, VETERANS HEALTH ADMINISTRATION
}

\begin{abstract}
Rehabilitation service providers in rural or underserved areas are often challenged in meeting the needs of their complex patients due to limited resources in their geographical area. Recruitment and retention of the rural clinical workforce are beset by the ongoing problems associated with limited continuing education opportunities, professional isolation, and the challenges inherent to coordinating rural community healthcare. People with disabilities who live in rural communities also face challenges accessing healthcare. Traveling long distances to a specialty clinic for necessary expertise is troublesome due to inadequate or unavailable transportation, disability specific limitations, and financial limitations. Distance and lack of access are just two threats to quality of care that now being addressed by the use of videoconferencing, information exchange, and other telecommunication technologies that facilitate telerehabilitation.

This white paper illustrates and summarizes clinical and vocational applications of telerehabilitation. We provide definitions related to the fields of telemedicine, telehealth, and telerehabilitation, and consider the impetus for telerehabilitation. We review the telerehabilitation literature for assistive technology applications; pressure ulcer prevention; virtual reality applications; speech-language pathology applications; seating and wheeled mobility applications; vocational rehabilitation applications; and cost-effectiveness. We then discuss external telerehabilitation influencers, such as the positions of professional organizations. Finally, we summarize clinical and policy issues in a limited context appropriate to the scope of this paper.
\end{abstract}

Keywords: Telerehabilitation; Telehealth; Telemedicine; Telepractice

\section{INTRODUCTION}

Telecommunications technologies are changing ways of thinking, acting, and communicating throughout the world and within healthcare. As in any technological area, healthcare and telecommunication definitions and language evolves with developing concepts and technological innovation. An example of this is the distinction between telehealth and telemedicine. Telehealth, one of the oldest areas of applied technology in medicine, refers to the use of electronic information and telecommunications technologies to support longdistance clinical health care, patient and professional health-related education, public health, and health administration. Telemedicine, one of the oldest areas of applied technology in medicine, is described as "the use of electronic information and communications technology to provide and support healthcare when distance separates the participants" (Hatzakis, Haselkorn, Williams, Turner, \& Nichol, 2003).

In an effort to clarify terminology and place telerehabilitation within the larger realm of telehealth, Jack
Winters (2002) provided a conceptual view of emerging models of telehealth with two major subsets: telemedicine (i.e. delivery of clinical services) and telehealthcare (i.e. management of disability and health). When Winters wrote his now classic article, telerehabilitation was an emerging field, positioned within both telemedicine and telehealthcare. There have subsequently been advances in the conduct of practice, particularly in the areas of physical therapy, occupational therapy, audiology, speech-language pathology, and neuropsychology. We thus propose that telerehabilitation warrants a separate and parallel identity under the "telehealth umbrella" alongside both telehealthcare and telemedicine.

There is a growing amount of literature on the use of technology for remote assessment and intervention in medicine (Bashshur, 2002) and rehabilitation (Lemaire, Boudrias, \& Greene, 2001; Torsney, 2003; Winters, 2002). Rehabilitation providers may not be aware of all telerehabilitation options available via innovative healthcare technologies. Additionally, they may not be 
fully aware of potential challenges inherent to technology application. These issues present an obstacle for the agency, individual provider, or consumer who would like to consider implementing telerehabilitation for a particular environment, purpose, or disability group that may not necessarily match available descriptions or what they know/currently access and use. For telerehabilitation to best benefit the end-user (an individual with a disability), all parties involved need to have access to the greatest possible set of available options to choose what will most likely work for the consumer and the environment in which they function. As telerehabilitation services continue to grow as a complement to traditional face-to-face clinical services, there is an increasing need to standardize appropriate clinical uses, reimbursement, and health care policy regarding the use of telerehabilitation.

\section{REVIEW OF SCIENCE}

\section{IMPETUS FOR TELEREHABILITATION}

Remote areas often experience shortages of professionals and technical resources crucial to the delivery of services related to specialized medical fields (Callas, Ricci, \& Caputo, 2000) . These shortages negatively affect both health care providers and patients. Rural providers are often isolated from medical advancements and technologies used in larger metropolitan centers. As a result, when an individual in a rural area needs an assessment and/or specific treatment, he or she may have to travel long distances to receive the specialized healthcare necessary to address their needs. Studies have reported that $50 \%$ of veterans travel more than 25 miles for healthcare (Randall, Kilpatrick, Pendergast, Jones, \& Vogel, 1987; Wollinksky, Coe, Mosely, \& Homan, 1985). Hatzakis (2001) conducted a study within the Veterans Health Administration which determined that veterans with multiple sclerosis have significant barriers to care as a result of their disability. Twenty percent of patients surveyed reported that parking, distance, or transportation difficulties significantly interfered with their receiving treatment. Furthermore, for individuals with sensation issues (e.g., spinal cord injury) prolonged sitting during travel can carry the potential risk of worsening a sore or decubitus ulcer. (Sabharwal, Mezaros, \& Duafenbach, 2001) For this reason, many individuals delay or avoid necessary treatment. While issues of access are clearly magnified in rural areas, mobility restrictions and accessibility problems also decrease the quality of healthcare for individuals located in urban areas (Hatzakis et al., 2003).

Appropriate selection and application of telerehabilitation technology may be conceptualized as a clinical reasoning task, since appropriate use of telerehabilitation requires assessment of individual needs and environmental factors; consideration of diagnostic issues; implementation of an intervention; and follow-up to determine efficacy. (A related white paper on infrastructure and technology provides more detail about the human and economic factors as well as the technology.) Human factors focus on system usability and designing system interfaces to optimize the users' ability to accomplish their tasks error-free in a reasonable time (Bashshur \& Lathan, 1999). Human factors is an applied science that takes research about human abilities, limitations, behaviors, and processes and uses this knowledge as a basis for the design of tools, products, and systems (Brennan \& Barker, 2008). This type of diagnostic reasoning has been conceptualized as a complex, dynamic process based on hypothesis testing (Stausberg \& Person, 1999) suggesting that clinical reasoning must match consumer experiences with clinical knowledge.

Since the late 1950's technologists and clinicians have investigated the use of advanced telecommunications and information technologies as a way of bridging the gap between individuals with specialized medical needs living in remote areas and the source of specialty care (Benschoter, Wittson, \& Ingham, 1965; Heinzelmann, Lugn, \& Kvedar, 2005; Kinsella, 1998). Again, telemedicine is described as "the use of electronic information and communications technology to provide and support healthcare when distance separates the participants" (Hatzakis et al., 2003). Telemedicine has been applied in many areas often first through smallerscale feasibility projects, and later in larger-scale clinical deployments that include cardiology (Cheung et al., 1998), dermatology (Lowitt et al., 1998), neurosurgery (Pareras \& Martin-Rogrigues, 1996), pathology (Ballis, 1997), radiology (Boland, 1998), oncology (Allen, 1997), and space exploration (Doarn, Nicogossian, \& Merrell, 1998). For further information, Krupinski et al. (2002) summarizes the state of the art for clinical applications in telemedicine and telehealth.

The rehabilitation field has been gradually integrating telecommunication tools into clinical practice. The benefits of using telerehabilitation include: 1) decreased travel between rural communities and specialized urban health centers; 2) better clinical support in local communities; 3) improved access to specialized services; 4) delivery of local health care in rural communities; 5) indirect educational benefits for remote clinicians who participate in teleconsultations; 6) reduced feelings of isolation for rural clinicians; 7) improved service stability in regions with high staff turnover; and 8) multimedia communication (Lemaire et al., 2001). Worldwide, the business of telehealth doubled from a $\$ 6.8$ million industry in 1997 to a $\$ 13.8$ million industry in 1998 (Savard, Borstad, Tkachuck, Lauderdale, \& Conroy, 2003). Experts have forecasted that by 2010 , at least fifteen percent of health care services worldwide will be provided via telehealth (Sinha, 2000). 


\section{SUMMARY OF TELEREHABILITATION LITERATURE}

\section{Assistive TECHNOLOGY APPLICATIONS}

Telerehabilitation is the application of telecommunication technology that provides distant support, assessment and intervention to individuals with disabilities (Ricker et al., 2002). Telerehabilitation offers many new opportunities to provide rehabilitation services in alternative ways and in different clinical settings (Cooper et al., 2001; Lemaire et al., 2001). Rory Cooper et al. (2001), from the University of Pittsburgh, have discussed the potential of rapid improvement in telecommunications technology to improve access to assistive technology (AT) services for people with disabilities. Assistive technology commonly refers to "...products, devices, or equipment, whether acquired commercially, modified or customized, that are used to maintain, increase or improve the functional capabilities of individuals with disabilities..." according to the definition proposed in the Assistive Technology Act of 1998 . Telerehabilitation offers a diversity of clinical applications. These include: 1) consultation by clinical rehabilitation engineers or specialized clinicians for seating and positioning; 2) the provision of assistive technology using simple Plain-old Telephone Service (POTS) videophones; 3) physicians and nurses performing pressure sore management using either higher-quality camera images or lower-quality images from interactive systems; 4) remote therapy using tools such as EMGcontrolled games for stroke rehabilitation or remote interactive story retelling for brain injury rehabilitation; 5) remote rehabilitation management or teleconsultation by physiatrists; and, 6) specialized clinicians for clinics using group videoconferencing systems over established telemedicine networks (Winters, 2002).

Burns et al. (1998), from Shepherd Center in Atlanta, described the experiences of a specialty hospital serving people with disabilities in exploring telerehabilitation to support assistive technology in the home. The article described four specific case studies to illustrate how telerehabilitation was used in relation to seating evaluation, home accessibility, setup of computer access systems, and augmentative communication device training. Each of the case studies used low-cost video telephone such as the AT\&T's Picasso Still Image Video Phone, and American Telecare Inc.'s Aviva 1010 and PTS-2 systems. All the devices transmitted simultaneous audio and video over standard telephone lines. The authors limited their choice to systems using the standard telephone lines because nearly everyone had access to a phone line in this research study. The respective objectives of the four case studies were the following: 1) to observe and assess the seating posture and the effectiveness of a patient's weight shift while seated in their recliner; 2) to evaluate an individual's strengths and limitations within the context of their existing bathroom structure and provide recommendations for adaptive equipment; 3) to provide recommendations for a 23 year old man with tetraplegia for a switch mounted system for computer access; and 4) to provide training to a 57 year old man with cerebral palsy on the use of his augmentative communication device. The case studies showed that telerehabilitation has great promise for expanding availability, accessibility, and affordability of services for people with disabilities. Each scenario, however, demonstrated limitations. During the evaluations, both the audio and video were not ideal for the conditions due to insufficient POTS line bandwidth, as well as the dim lighting for video capture. The authors found that the clinical staff members who provided the respective consults needed more time to familiarize themselves with the technology before mailing it to the clients.

In recent years, the availability of high-speed Internet in the home has grown tremendously. Internet-based systems designed as plug-play appliances that do not require a computer are becoming more common. However, POTS devices are still the most prevalent resource for providing home tele-services. Therefore, while considering advanced internet-based applications, the developers and manufacturers of telerehabilitation technologies must also consider compatibility with POTS lines.

\section{PreVENTION OF PRESSURE UlCERS}

Several studies have examined the feasibility of preventing pressure sores and promoting pressure relieving activities (Beach, Goodall, \& Miller, 2000; Mathewson, Adkins, \& Jones, 2000; Mathewson, Adkins, Lenyoun, Schmidt, \& Jones, 1999; Roth, Reid, Puckett, \& Concannon, 1999; Soopramanien, Pain, Stainthrope, Menarini, \& Ventura, 2005; Vesmarovish, Walker, Hauber, Temkin, \& Burns, 1999). Philips et al. (1998), from Emory University, studied the use of a telerehabilitation intervention to promote skin and other self-care activities for people with spinal cord injuries. This was a noncontrolled clinical series where researchers used a video phone that would transmit video and still images over a standard telephone line. The overall impression of the eleven clients was noted to be positive, however, there were some problems reported with equipment, skin inspection, and safety. Diagnosing skin conditions through verbal description alone was not reliable, and the remote clinician wanted to be in the same room face-toface to examine the skin.

In another study, Philips et al. (1999), conducted a non-randomized controlled prospective trial with thirtyseven patients. The authors examined injured spinal cord patients after hospital discharge via three different 
approaches (i.e., telephone, video, and standard care) to determine which approach was associated with the lowest incidence of pressure ulcers and the fewest rehospitalizations. The results from this pilot study indicated that the video group reported the highest number of ulcers and did so accurately, while the standard care reported the lowest number of ulcers. A larger sample is required for a more comprehensive analysis. The authors advised that prior to discharge patients will require additional training on how to set-up and use the equipment, to minimize the costs of clinician and technician travel to the patients' homes.

\section{VIRTUAL REALITY APPLICATIONS}

Virtual Reality (VR) is becoming a practical, affordable technology for the practice of clinical medicine. Modern high fidelity VR systems have practical applications in areas ranging from psychiatry to intervention and rehabilitation (Bergeron, 2003). VRs' capacity to allow for creation and control of 3-dimensional built environments offers clinical assessment and rehabilitation options that are not available with traditional methods (Schultheis \& Rizzo, 2002). Virtualized reality and 3D reconstruction technology provided an effective means investigating the architectural features of a built environment without an expert visiting the site for individuals who use a wheeled mobility device (Kim \& Brienza, 2006; Kim, Brienza, Lynch, Cooper, \& Boninger, 2008). Harrison et al. (2002) applied two virtual environments to the assessment and training of inexperienced powered wheelchair users and demonstrated that the two virtual environments represent a potentially useful means of assessing and training novice powered wheelchair users.

The University of Medicine and Dentistry of New Jersey designed the remote console (ReCon) (Lewis, Boian, Burdea, \& Deutsch, 2005), a telerehabilitation system designed to provide therapists, at a remote location, the tools necessary to oversee patient's rehabilitation session in real-time. This system provides the therapist with threedimensional representations of patients' movements, VR-based exercise progress, and performance updates while the patient is exercising. During the session, the therapist evaluates the patient's performance and either modifies the current exercise, or sets up the next one. The remote therapist is also provided with tools for audio and video communication with the local site and chat communication with the local therapist. Researchers conducted both usability and evaluation studies to refine the system (Lewis et al., 2005; Lewis, Deutsch, \& Burdea, 2006). Trepagnier (1999) described the value of VR systems for the investigation and rehabilitation of cognitive and perceptual impairments and discussed current and political applications of VR technology. The following were the neurorehabilitation issues: (1) attention and the reduction of distraction; (2) assessment and remediation of executive function deficits; (3) investigation of impairments of coordinated movement; (4) study and rehabilitation of aphasia and other severe disorders of language; (5) task presentation for functional imaging studies of the brain; and (6) the measurement of mental load in the operation of assistive technology.

\section{SPEECH-LANGUAGE PATHOLOGY APPLICATIONS}

Speech language pathology (SLP) and audiology are clinical services related to the identification, assessment and management of hearing and communication disorders. SLP is ideally suited for telerehabilitation as the client-clinician interaction is primarily visual and verbal. Many of the assessment and treatment materials can be administered via computerized programs. The Mayo Clinic was one of the earliest to incorporate teleconsultations into SLP which provided a viable alternative to the traditional face-to-face assessment (Duffy, Werven, \& Aronson, 1997). Researchers at the National Rehabilitation Hospital in Washington, D.C. developed a custom software package called RESPECT (REmote SPEech language and Cognitive Treatment) which investigates the role of interactive data sharing during teleSLP cognitive communicative treatment. RESPECT augments and extends therapeutic interaction with the following capabilities: virtual desktop, real time shared interaction, work processing documents, scanned workbook pages, computer applications, digital drawing whiteboards, and combined audio/video conferencing where the clinician controls clients' system. Research has established the validity of the using the system in a story retelling between face-to-face, or in-person assessment, and remote telerehabilitation sessions, with a sample of 40 subjects with brain injury (Georgeadis, Brennan, Barker, \& Baron, 2004). In addition to equivalent performance between settings, a high level of acceptance of telerehabilitation technology was found regardless of subjects' age, educational levels and technology backgrounds.

The Telerehabilitation Research Unit at the University of Queensland (http://www.uq.edu.au/telerehabilitation/) is another pioneering institution. Their research involves the assessment and treatment for individuals with acquired neurological speech and language disorders. One of their many research projects reported significant improvements in speech for individuals diagnosed with Parkinson's Disease using the Lee Silverman Voice Treatment via the internet (Theodoros, Constantinescu, \& Russell). Other examples include voice therapy at Tripler Army Base in Hawaii (Mashima et al., 2003); remote dysphagia evaluations (Georges, Potter, \& Belz, 2006; Perlman \& Witthawaskul, 2002); and augmentative and alternative communication evaluations (McKinlay, Beattie, Arnott, \& Hine, 1995). 


\section{SEATING AND WHEELED MOBILITY APPLICATIONS}

Several studies analyzed the use of telerehabilitation in the field of seating and mobility. Related to seating and positioning, telerehabilitation has the potential to provide evaluation, treatment intervention, and follow-up as needed in the home of the client or at a local clinic (Philips et al., 1998). Assessment in the home of the client is especially important considering that the use of assistive technologies (e.g., wheelchairs) is only as effective as the ability of the individual to use it in their own environment. Research has demonstrated that the use of telerehabilitation can help promote community re-entry and improve the quality of life of the individual (Philips et al., 1998). The Glenrose Rehabilitation Hospital in Alberta Canada presented a study design and protocol to evaluate the effectiveness and efficiency of using telehealth to provide seating assessment and intervention by comparing groups of clients in three conditions: 1) clients residing in Capital Health assessed in-person; 2) clients from out-of-region assessed inperson; and 3) clients from out-of-region assessed by telehealth whereby results have not been published (Liu \& Barlow, 2005). A qualitative case study was conducted among rehabilitation professionals for implementing and planning a telehealth seating clinic (Khoja, Casebeer, \& Young, 2005). The study showed that when implementing such a clinic, the involvement of multidisciplinary teams and proper visualization and communication between participants is essential.

Malagodi et al. (1998) compared videoconferencing using ordinary POTS lines with videoconferencing using Integrated Services Digital Network (ISDN) lines. Over six months, an occupational therapist completed eight seating and wheelchair mobility evaluations. Four clients were evaluated via videoconferencing using the POTS line, and four clients using an ISDN line. Despite challenges presented via the technology used the time (i.e. lower quality video afforded by POTS lines, lower data communications rate led to longer still picture transfer times and jerkier video images than was achieved with the ISDN connections) the primary condition and major problem were correctly identified in all cases. This work showed that with advancements in telecommunication technology, telerehabilitation systems could have the potential to greatly affect how services are delivered and to carefully determine the best and most appropriate AT device for the client.

Cooper et al. (2002) compared the type of wheelchair the person actually uses, to the wheelchair recommended via telerehabilitation and in-person assessments.

Clinicians utilizing telerehabilitation demonstrated a high level of agreement in recommending the same basic type of wheelchair that subjects already owned, showing a high level of agreement in the consistency of wheelchair recommendation. Therefore, telerehabilitation is a potentially useful tool for wheelchair recommendation.

\section{VOCATIONAL REHABILITATION APPLICATIONS}

Individuals with significant disabilities experience challenges to achieving vocational goals and employment outcomes. With current census data indicating that the employment rate of working age people with disabilities is 37.5 percent in 2004, access to effective rehabilitation resources and services is of paramount importance (Rehabilitation Research and Training Center on Disability Demographics and Statistics, 2005). Persons with disabilities may experience a complex array of functional limitations that, along with external barriers (accessibility, attitudinal, lack of resources), impact their ability to perform effectively in vocational training and employment settings, as well as in social, recreational and independent community living. Vocational and independent living rehabilitation services have evolved and have been demonstrated through research and practice to be more effective in achieving employment and community independence outcomes. However, due to limited availability of services, and cost and accessibility challenges, not everyone with a disability has access to resources and services that have demonstrated efficacy and that are specific to their interests and needs. Telerehabilitation may provide a mechanism to enable individuals with disabilities to gain access to effective vocational rehabilitation services, regardless of limitation imposed by geography and local resource capabilities.

This section will focus on telerehabilitation applied to the needs of persons with disabilities in the realm of employment. With respect to employment, this involves vocational assessment; preparation for employment (such as education and training, development of pre-vocational skills and competencies); seeking and obtaining employment (developing job seeking skills, participating in peer or group job search clubs); maintaining employment (on and off site job coaching and supported employment); and monitoring and follow-up (ongoing assessment of performance and consultation in meeting new job demands or addressing obstacles).

Despite the potential and need for telerehabilitation in vocational rehabilitation, very few applications have been reported. Trepagnier, Noiseux and Glenshaw (1999) described the results of a series of focus groups that investigated consumer acceptance of remote monitoring identification of roles for video technology in supported employment. The focus groups provided access to technologies and solicited input and feedback. Results indicated that video technologies were thought to have potential as a medium of vocational support. Specific recommended applications included job development, client monitoring and real time support, and reassurance and instruction/cueing. The video technology was thought to be more useful than audio only in working with individuals with cognitive impairments, and was viewed by the respondents as providing greater flexibility 
in coverage for vocational support providers. Potential drawbacks included employer technophobia and privacy concerns. Several researchers have indicated the value of telemedicine or telerehabilitation in enhancing the delivery of more traditional rehabilitation services. Cole and Matthews (1999) reported that telemedicine enables the delivery of cognitive prosthetic services by a therapist in the home. Ricker et al. (2002) reported survey results that individuals with acquired brain injury expressed strong interest in a variety of potential Internet-based rehabilitation services such as memory, attention, problem solving and activities of daily living assistance.

In a study that was designed to test how telerehabilitation technology might be accepted and effectively utilized by persons with cognitive disabilities, McCue, Lang, Bates, \& Germek, (2003) assessed the effect of high and low bandwidth videoconferencing on persons with known attention, perceptual processing and language comprehension problems. This was done through administration of a battery of psychometric instruments via video teleconferencing in face-to-face, low and high bandwidth conditions.

Results revealed that in a sample of 15 individuals with cognitive disability, there was a significant difference between face-to-face assessment and low and high bandwidth on only a complex memory task involving list learning. There was no difference on tests of understanding oral directions, repeating number series, and on tasks involving controlled fluency. These findings suggest that despite information processing limitations in this population and apparent fluctuations in audio and video quality, televideoconferencing (including low bandwidth applications) is an effective mechanism for communication. Findings supported the use of telerehabilitation as a potential means of support and rehabilitation intervention with individuals with cognitive disabilities.

Brennan et al. (2004) conducted a study to measure performance by forty brain-injured subjects with medical diagnoses of stroke or traumatic brain injury, on a standardized Speech-Language Pathology evaluation conducted in both face-to-face and videoconferencebased telerehabilitation settings. The Story Retelling Procedure (SRP), which measured connected language production and comprehension of spoken narratives, was administered to each subject in both settings. The objectives of the study were to: (1) compare communication as measured by the SRP between experimental settings; and (2) determine if variables such as age, education, technology experience or gender had an effect on performance between settings. The results concluded that no significant difference was found between SRP performance measured in the two settings. The variables of interest (i.e., age, education, technology experience, and gender) did not significantly affect the difference between performances in the two settings. Additionally, the subjects reported a high level of acceptance of using the videoconferencing system when asked if they would use it again to talk with their clinician.
Chase and Danilko (2005) conducted a pilot study using remote technologies to provide remote coaching aimed at assessing the feasibility of participant's vocational goals. Remote technology used to deliver services included phone calls, teleconferencing, email and web based application sharing enabling the participant to see the coach's computer screen. Coaching focused on helping participants to clarify their goals, contact experts in the community to learn more about the demands of their target job, evaluate their skills against the demands of the job, and map out specific action steps to pursue the "best fit" jobs. A concise feasibility plan summarizing this information was created through the coaching sessions and delivered to the participants and their counselors. Each participant met key benchmarks through the program (such as interviewing a community expert) in a very tight time frame. Participants were able to achieve specific benchmarks toward determining the feasibility of their employment goals. The significant benchmarks reached the overall ratings of the project by participants, and the use of technology to deliver distance services were generally very positive. By contrast, change in pre and post ratings of clarity of employment goals, understanding of job demands, etc. from participant and counselor surveys were minimal, or even at times negative. The authors speculated that increased information about the actual demands of the target job gleaned from the remote coaching might have led to greater feelings of uncertainty.

McCue, Pramuka, Smathers, Germek, Muncert and Lang (2004) conducted a pilot study to evaluate the potential of a number of remotely implemented telecommunication technologies to individuals with cognitive disabilities to succeed in the academic or work environment. In this particular study, several telecommunication technologies were implemented through a wireless Phone/PDA by persons with cognitive disabilities, accompanied by face-to-face and remote training interventions, and tested. The protocol evaluated cellular phone technology (wireless phone and text messaging) and Internet technology (instant messaging, file sharing and e-mail). Results revealed modest gains from the technology. Barriers, including technical difficulties and an overestimation of the subjects' level of functioning, were considered to limit overall success. Authors reported that that distance support technologies had a positive effect on everyday functioning. In particular, text and instant messaging showed the greatest potential for providing rehabilitation supports "in-vivo" to persons with cognitive disabilities (McCue et al., 2004).

In summary, while there have been few studies published on the use and efficacy of telerehabilitation applied to vocational rehabilitation, the pilot studies that exist demonstrate potential for broader application of remote technologies to meet the vocational rehabilitation and employment support needs of persons with disabilities. 


\section{COST-EFFECTIVENESS}

Telerehabilitation may not only provide cost-effective treatment options to patients, but may also permit more convenient training of healthcare professionals (Callas et al., 2000; Delaney et al., 2002; Grigsby, Schlenker, Kaehny, Shaughmessy, \& Sandberg, 1995; Jennett et al., 2000; Lemaire et al., 2001; Taylor, 1998; Zollo, Kienzle, Henshaw, Crist, \& Wakefield, 1999). Cost effectiveness of "tele" projects is being studied as there considerable controversy about how to measure the cost of these efforts (Kitt \& Clayton, 2002; Mair, Haycox, May, \& Williams, 2000; Ohinamaa \& Hailey, 2002; P. S. Whitten $\&$ Mair, 2000). Critical reviews of the cost-effectiveness and cost-benefit literature have been published (Gamble, Savage, \& Icenogle, 2004; Hakansson \& Gavelin, 2000; Mair et al., 2000; P. Whitten, Kingsley, \& Grigsby, 2000). Although these studies provide evidence that telemedicine may be cost-effective, the cost of providing care for that facility increases as a result of additional costs of equipment, transmission lines, additional personnel, and program administrative. The question of cost-effectiveness remains unanswered for most of the telemedical services that are developed worldwide because of objective and subjective problems (Holle \& Zahlmann, 1999).

\section{CLINICAL AND POLICY ISSUES}

Telerehabilitation has and will continue to encounter considerable resistance or barriers as it moves from the perimeter to the mainstream of healthcare over the coming years. The intent of this white paper is not to address in-depth discussion on policy issues but rather provide an overview of current clinical concerns associated with telerehabilitation and assistive technology. Some of the questions that have arisen include:

- How and who is going to pay for reimbursement?

-Who is qualified to perform such assessments, treatment intervention, and routine service delivery?

- How is quality assessed?

Quality must be defined based on the technology (i.e. signals and data) and the clinical quality of the services (i.e. outcomes). Additional concerns are issues of confidentiality; how remote service delivery fits into rehabilitation professionals' codes of ethics respectively; the paucity of scientific evidence; the lack of standards and guidelines; and questions regarding credentialing and interstate licensing regulations.

Telerehabilitation is advancing at a time when health insurance providers are reimbursing less for most services and products. Currently, telerehabilitation services are not expected to be fully covered because full coverage for most health services is no longer the norm. On May 22, 2008, Representative C. Michael Thompson and fourteen other co-sponsors introduced H.R. 6163, the Medicare Telehealth Enhancement Act of 2008. The proposed bill would have:

- Expanded Medicare reimbursement for telemedicine into urban areas;

- Authorized a study on store-and-forward telemedicine;

- Expanded originating sites for Medicare to include skilled nursing facilities, dialysis centers and community mental health centers;

- Added PTs, OTs, and SLPs as telehealth providers under Medicare; and

- Encouraged the adoption of reciprocity agreements for licensure across state lines.

Certain parts of this proposed bill were included in a comprehensive Medicare bill, H.R.6331, but the reimbursement for the rehabilitation providers was not. Unfortunately, the legislative process is often an incremental one, and it is very common to get a little bit of what was wanted and thus need to be proactive in upcoming years.

Medicare payment of telemedicine and telehealth services is divided into three areas: 1 ) remote patient face-to-face services seen via live videoconferencing ; 2) non face-to-face services that can be conducted either through live video-conferencing or via store and forward telecommunication service and 3) home telehealth services. In order for telerehabilitation to be accepted for reimbursement by third party payers, government officials want to see sound scientific research. Research in telerehabilitation is in its infancy, with only a handful of equivalence trials. As of 2006, most peer-reviewed research articles are case reports of pilot programs or of testing new equipment. Rehabilitation researchers need to conduct many more controlled experiments and present the evidence to clinicians and payers that it is clinically effective. Susan Palsbo (2004) assessed current payment practice for telerehabilitation in state Medicaid programs which revealed that only four states at that time were providing reimbursement (Hawaii, Louisiana, Minnesota, and Nebraska). For more information, the Center for Telemedicine Law (2003b) prepared an in-depth written report on the current state of telemedicine reimbursement for the Office for the Advancement of Telehealth.

Another issue of importance is licensure. Telerehabilitation challenges the traditional practice involving a face-to-face encounter between rehabilitation professional and patient. Telerehabilitation breaks the physical link and complicates decisions about where a telerehabilitation clinician should be licensed if the clinician and patient are located in different states (Gilbert, 1995; Granade, 1995). Rehabilitation professionals are typically required to be licensed by the state in which they are practicing their profession. This creates a problem and a dilemma when telerehabilitation services are delivered to a patient across state lines. However, telerehabilitation is not unique, as all 
telemedicine applications face similar challenges. State practice acts have not outlawed telemedicine, as these laws were passed before any state legislator could imagine its development. Obtaining a professional state license requires the completion of paperwork and the payment of upfront costs and renewal fees. Multiple state licenses can involve considerable time and paperwork (Collins \& Charboneau, 1993). Practitioners need to continue conducting and researching the application of telerehabilitation. They also need to present their findings to their respective professional associations and advocate to government officials at both the state and federal levels. For more information, the Center for Telemedicine Law (2003a) prepared an in-depth written report on the current state of telemedicine licensure for the Office for the Advancement of Telehealth.

\section{PROFESSIONAL ORGANIZATIONS' VIEWS ON TELEREHABILITATION}

Professional organizations such as the American Speech-Language and Hearing Association (ASHA), American Occupational Therapy Association (AOTA), American Physical Therapy Association (APTA), and the Commission on Rehabilitation Counselor Certification (CRCC) have different views on what constitutes telerehabilitation. Telerehabilitation is a relatively new application, and the degree to which each organization has acknowledged and supported telerehabilitation varies, as does their level of activity and involvement in telehealth activities. There needs to be increased collaboration and cohesiveness among professional rehabilitation associations concerning telerehabilitation.

ASHA uses the term "telepractice" to refer to "the application of telecommunications technology to deliver professional services at a distance" (American SpeechLanguage-Hearing Association, 2005). Since 1998, ASHA has studied the potential impact of telepractice on speech-language pathologists (SLPs) and audiologists and the individuals they serve (American SpeechLanguage-Hearing Association, 1998). ASHA has published the most abundant amount of literature related to the field of telehealth. The first documented use of distance programs in SLP was through a grant program in the mid-1970s at the Birmingham Veterans Administration Hospital to explore "tele-communicology" as a potential solution to serving patients in remote locations (Vaughn, 1976). The National Rehabilitation Hospital in Washington D.C. and the University of Queensland in Australia have emerged as two of the leaders in studying telerehabilitation activity related to speech-language pathology and audiology. ASHA has published a formal position statement, technical reports (American SpeechLanguage-Hearing Association, 2005) and issue briefs (American Speech-Language-Hearing Association, 1998) that summarize evidence to date about the use of telepractice in SLP and audiology and discusses future directions and research.

APTA has released a Board of Directors Position on telehealth and reference is made in the position on definitions of telehealth and electronic communications (American Physical Therapy Association Government Affairs). There are also articles explaining how physical therapists are using telehealth to overcome barriers of distance and time. Kathy Lewis, Past-President of the APTA Section on Health Policy and Administration's Technology Special Interest Group, observed that many forms of technology use are increasing in physical therapy practice. She also stated, "It (technology) can increase our practice scope, and it is a tool. I hope that more PTs will take an interest in technology and how it can help us improve the care we provide."

AOTA has also published a position paper on telerehabilitation which presents both their stance and the literature supporting methods of service delivery for evaluation (Shaw, Dreyer, Dreyer, \& Wittman, 2001); intervention (Vesmarovish et al., 1999); consultation (Wakeford, 2002); education; and the supervision of students and other personnel (Hubbard, 2000). Telerehabilitation, as defined by the AOTA position paper, is the clinical application of consultative, preventative, diagnostic, and therapeutic services via two-way interactive telecommunication technology (Wakeford, Whitman, White, \& Schmeler, 2005). The Canadian Association of Occupational Therapists) is another professional association that has a position statement about telehealth and tele-occupational therapy. CAOT recognizes the ongoing development of tele-occupational therapy, which will promote opportunities for effective, efficient, and accessible occupational therapy services, education and resources to all Canadians.

In vocational rehabilitation and rehabilitation counseling, the Commission on Rehabilitation Counselor Certification (CRCC) is the independent credentialing body for the profession. While CRCC has not established a formal position on telerehabilitation, it is currently proposing a significant revision to the ethics code (a joint code developed jointly by CRCC and the two major professional organizations representing rehabilitation counseling, the National Rehabilitation Counseling Association - NRCA, and the American Rehabilitation Counseling Association - ARCA). The draft revisions contain considerable new practice guidelines regarding the use of technology in assessment and counseling. Among other areas, the draft guidelines state that rehabilitation counselors are held to the same level of expected behavior as defined by the ethics code regardless of the technology used (e.g., cellular phones, email, facsimile, video, audio, audiovisual) or its application (e.g., assessment, research, data storage). It provides detailed practice guidelines on issues related to problematic use of the Internet, privacy, confidentiality and security. 


\section{SUMMARY AND PRELIMINARY FINDINGS}

The rapid improvements in telecommunications technology have the potential to improve the delivery of services to people with disabilities and those who are elderly. Transmission of voice, image, and data could provide a means for experts in wheeled mobility and in other rehabilitation fields to provide consultation to other healthcare professionals and consumers (Cooper et al., 2001).

Telerehabilitation may prove to be a promising alternative for individuals who otherwise would have no option but to travel long distances to receive services they need. Researchers have shown that telerehabilitation can improve quality of life and lead to more efficient use of health care resources (Burns et al., 1998; Krupinksi et al., 2002). Bashshur (2001) investigated the cost/ benefit ratio of using a technology like telemedicine. While technological advances have produced significant improvements in healthcare, ironically, it is technology itself that has played a role in the rising costs of care. However, the authors believe that telerehabilitation technology can, in fact, improve access and enable patients to receive appropriate care in their own homes or at nearby healthcare facilities and result in improved quality of care. This care would be provided at local health facilities by their current health providers, but with the aid of remote telerehabilitation consultant specialists. Given the apparent success of telerehabilitation in this scenario, such an application could reduce the need of specialists to travel to remote locations, without compromising the quality of care that clients receive. This study further explains that the cost of care at local facilities is likely to be less than that at highly specialized care centers (Bashshur, 2001).

Despite encouraging studies demonstrating the feasibility of telerehabilitation, their applications are restricted by limited reimbursement for services. Medicare generally has not been receptive towards increasing telerehabilitation reimbursement based on the fact that "there is very little published peer-reviewed scientific data available on when telemedicine use is medically appropriate" and on the effectiveness of telerehabilitation (Hatzakis et al., 2003).

In response, University of Pittsburgh researchers and clinicians have collaborated to investigate the effectiveness of telerehabilitation interventions and explore its potential as a clinical tool to address the gaps and improve quality of care. One of the research tasks of the Rehabilitation Engineering Research Center on Telerehabilitation (RERC-TR) is the evaluation of remote wheelchair prescription. The need for wheeled mobility devices is increasing as our population is aging and surviving trauma and disease. The availability of practitioners with specific expertise in this area is limited, especially in rural areas. People are isolated from rehabilitation services due to geography or physical limitations whereby large distances require long travel times increasing costs and other burdens. The purpose of this project is to determine the effectiveness of using a secure telerehabilitation consultation model for procuring an appropriate wheeled mobility and seating device via the high speed internet.

Outcome studies in the field of telerehabilitation certainly start in the laboratory or university setting, but they extend out to the actual settings and to the patients that will ultimately benefit the most (i.e. rural or underserved communities). Researchers have taken the custom secure Internet Protocol based videoconferencing system developed by the RERC-TR and installed it within five rural hospitals located at least 100 miles away from Pittsburgh. Before launching the system, feasibility studies were conducted with one of the remote sites to test the camera technology associated with the videoconferencing. It was determined that a basic USB web-camera with up to 8 megapixel, autofocus lens system, microphone with Rightsound technology, and up to 30 frames per second video met our needs. A second camera was installed to remotely control the ability to pan, tilt, and optical zoom to assist with the teleconsultation. A comparative approach against a referenced procedure or a predefined standard such as the one implemented by the Center for Assistive Technology at the University of Pittsburgh Medical Center (CAT-UPMC) was conducted (Schein, Schmeler, Brienza, Saptono, \& Parmanto, 2008). Many so called evaluation studies ignore this basic requirement and therefore often end up with inconclusive results. Current accuracy and outcome studies are being conducted and results will be finalized.

A second study focuses upon job coaching and involves activity recognition technologies using video and accelerometers to monitor specific work behaviors and, ultimately, to deliver cues and instruction in response to problem behaviors. The system is trained using accelerometer data from a given task being performed. A model is then developed to automatically recognize components of the task (or errors) during real time task performance and provide task guidance feedback to a client as needed via headphone.

Pilot usability studies revealed that results of video captured and analyzed by a machine learning technique used to automatically identify features in the person's movement patterns of a routine fast food work task (e.g. flipping, salting, placing on or taking burgers off the grill) indicated that none of the complex movements were mislabeled and a set of common errors were identified and labeled. The activity recognition was then tested using accelerometers (one on each hand, and one on each forearm) with results being essentially equivalent to video data. Thus, initial work has yielded a model that is able to correctly identify component tasks for multiple subjects, using video or accelerometers. A user interface 
that is able to utilize logged data to recognize and deliver task guidance cues remotely to the user in real time was developed. Additional investigation including broadening the subject base and developing an interface to share a summary of the data (e.g. success rate, types of errors) with a job coach or other support person is being undertaken (McCue, Hodgins, LoPresti, \& Bargteil, 2008).

As telerehabilitation moves beyond basic POTS and ISDN-based videoconferencing systems, rehabilitation clinicians and engineers have the increasing ability to upload and download data farther and wider. Yet, it is important to recognize that while the technology may be new, the rehabilitation services and care management systems are still the same. Advanced technologies including broad bandwidth Internet connections, webbased videoconferencing, and multimedia databases have potential to further enhance telerehabilitation capabilities. Telerehabilitation has the potential to both increase the availability of specialty clinical services in geographically remote communities and reduce service delivery costs associated with travel and time. Telerehabilitation's full potential will be only realized by researching and developing telecommunication technology that delivers both effective and efficient care to patients in all areas. Ultimately, the widespread adoption of telerehabilitation will require more than just lower cost technology information and access. These innovative strategies will need to be embraced by rehabilitation practitioners in their respective fields and those who pay for their services.

\section{REFERENCES}

Allen, A. (1997). Practising oncology via telemedicine. Journal of Telemedicine and Telecare, 3, 63-70.

American Physical Therapy Association Government Affairs. Telehealth - Definitions and Guidelines BOD G03-06-09-19 (No. (Program 19) [Retitled: Telehealth; Amended BOD G03-03-07-12; Initial BOD 11-01-28-70]). Alexandria: American Physical Therapy Association.

American Speech-Language-Hearing Association. (1998). Telehealth issues brief. Rockville, MD.

American Speech-Language-Hearing Association. (2005). Speech-Language Pathologists Providing Clinical Services via Telepractice: Technical Report from www. asha.org/policy

Ballis, U. J. (1997). Telemedicine and pathology. Clinics in Laboratory Medicine 7, 245-261.

Bashshur, R. L. (2001). Where are we in telemedicine/ telehealth, and where we go from here. Telemedicine Journal and e-Health, 7(4).

Bashshur, R. L. (2002). Telemedicine and health care. Telemedicine Journal and e-Health, 8(1), 5-12.

Bashshur, R. L., \& Lathan, C. E. (1999). Editorial: Human factors in telemedicine. Telemedicine Journal, 5(2), 127128.

Beach, M., Goodall, I., \& Miller, P. (2000). Evaluating telemedicine for minor injuries units. Journal of Telemedicine and Telecare, 6(Suppl 1), 90-92.

Benschoter, R. A., Wittson, C. L., \& Ingham, C. G. (1965). Teaching and consultation by television. I. Closed-circuit collaboration. Hospital and Community Psychiatry, 16, 99-100.

Bergeron, B. (2003). Virtual reality applications in clinical medicine. Journal of Medical Practice Management, 18(4), 211-215.

Boland, G. (1998). Telemedicine in practice. Seminars in Nuclear Medicine, 28, 145-157.

Brennan, D. M., \& Barker, L. M. (2008). Human factors in the development and implementation of telerehabilitation systems. Journal of Telemedicine and Telecare, 14(2), 83-87.

Brennan, D. M., Georgeadis, A. C., Baron, C. R., \& Barker, L. M. (2004). The effect of videoconference-based telerehabilitation on story retelling performance by brain-injured subjects and its implications for remote speech-language therapy. Telemedicine Journal and e-health, 10(2), 147-154.

Burns, R. B., Crislip, D., Daviou, P., Temkin, A., Vesmarovish, S., Anshutz, J., et al. (1998). Using telerehabilitation to support assistive technology. Assistive Technology, 10(2), 126-133.

Callas, P. W., Ricci, M. A., \& Caputo, M. P. (2000). Improved rural provider access to continuing medical education through interactive videoconferencing. Telemedicine Journal and e-Health, 6(4), 393-399.

Center for Telemedicine Law. (2003a). Telemedicine Licensure Report. Washington, D.C.: Office for the Advancement of Telehealth.

Center for Telemedicine Law. (2003b). Telemedicine Reimbursement Report. Washington, D.C.: Office for the Advancement of Telehealth. 
Chase, S., \& Danilko, N. (2005). A Model of employment feasibility planning. Technical Report 04-NTSTM215-04: Center of Excellence for Remote and Medically Underserved Areas, July 19.

Cheung, S. T., Davies, R. F., Smith, K., Marsh, R., Sherrard, H., \& Keon, W. J. (1998). The Ottawa telehealth project. Telemedicine Journal, 4, 259-266.

Cole, E., \& Matthews, M. K. (1999). Cognitive prosthetics and telerehabilitation: Approaches for the rehabilitation of mild brain injuries. In J. Murner \& T.M. Ettlin, (Eds.), HSW - Distorsion \& Leichte Traumatische Himverletzung Behandlungskonzepte. Basel, Switzerland: Institute for Cognitive Prosthetics.

Collins, J. M., \& Charboneau, W. (1993). Long distance procedures: Legal, licensing, and reimbursement issues. Paper presented at the Mayo Telemedicine Symposium, Rochester.

Cooper, R., Fitzgerald, S., Boninger, M., Brienza, D., Shapcott, N., \& Cooper, R. A. (2001). Prolog to Telerehabilitation: expanding access to rehabilitation expertise. Paper presented at the Proceedings of the IEEE.

Cooper, R., Fitzgerald, S., Boninger, M., Cooper, R. A., Shapcott, N., Cohen, L., et al. (2002). Using telerehabilitation to aid in selecting a wheelchair. Paper presented at the Rehabilitation Engineering \& Assistive Technology Society of North America, Minneapolis, Minnesota.

Delaney, G., Lim, S. E., Sar, L., Yang, S. C., Strumberg, J. P., \& Khadra, M. H. (2002). Challenges to rural medicine education: a student perspective. Australian Journal of Rural Health, 10(3), 168-172.

Doarn, C. R., Nicogossian, A. E., \& Merrell, R. C. (1998). Applications of telemedicine in the United States Space Program. Telemedicine Journal, 4, 19-30.

Duffy, J. R., Werven, G. W., \& Aronson, A. E. (1997). Telemedicine and diagnosis of speech and language disorders. Mayo Clinic Proceedings, 72(11), 1116-1122.

Gamble, J. E., Savage, G. T., \& Icenogle, M. L. (2004). Value-chain analysis of a rural health program: toward understanding the cost benefit of telemedicine applications. Hosp Top, 82(1), 10-17.

Georgeadis, A. C., Brennan, D. M., Barker, L. N., \& Baron, C. R. (2004). Telerehabilitation and its effect on story retelling by adults with neurogenic communication disorders. Aphasiology, 18, 639-652.

Georges, J., Potter, K., \& Belz, N. (2006). Telepractice program for dysphagia: Urban and rural perspectives from Kansas. The ASHA Leader, 11(15), 12.

Gilbert, F. (1995). Selected legal issues in the use of community health information networks. The Journal of Health Information and Management Systems Society, 9(2), 43-51.

Granade, P. (1995). Malpractice issues in the practice of telemedicine. Telemedicine Journal, 1(2), 87-89.

Grigsby, J., Schlenker, R., Kaehny, M., Shaughmessy, P., \& Sandberg, E. (1995). Analytic framework for evaluation of telemedicine. Telemedicine Journal, 1, 31-39.
Hakansson, S., \& Gavelin, C. (2000). What do we really know about the cost-effectiveness of telemedicine? Journal of Telemedicine and Telecare, 6(Suppl 1), 133136.

Harrison, A., Derwent, G., Enticknap, A., Rose, F. D., \& Attree, E. A. (2002). The role of virtual reality technology in the assessment and training of inexperienced power wheelchair users. Disability and Rehabilitation, 24(11), 599-606.

Hatzakis, M., Haselkorn, J., Williams-Avery, B. K., \& Rodriguez, A. (2001). Proportion of veterans with multiple sclerosis receiving pharmacology therapy for fatigue. International Journal of MS Care, 3(2), 4.

Hatzakis, M., Haselkorn, J., Williams, R., Turner, A., \& Nichol, P. (2003). Telemedicine and the delivery of health services to veterans with multiple sclerosis. Journal of Rehabilitation Research and Development, 40(3), 265-282.

Heinzelmann, P. J., Lugn, N. E., \& Kvedar, J. C. (2005). Telemedicine in the future. Journal of Telemedicine and Telecare, 11, 384-390.

Holle, R., \& Zahlmann, G. (1999). Evaluation of Telemedical Services. IEEE Transactions on Information Technology in Biomedicine, 3(2), 84-91.

Hubbard, S. (2000). A case example of remote supervision. OT Practice, 5(24), 16-18.

Jennett, P. A., Nijssen-Jordan, C., Wiggs, B., Gao, M., Person, V., Andruchuk, K., et al. (2000). Telelearning in a partnership between a university faculty and a regional health authority: benefits, challenges and strategies. Journal of Telemedicine and Telecare, 6(2), 32-35.

Khoja, S., Casebeer, A., \& Young, S. (2005). Role of telehealth in seating clinics: a case study of learners' perspectives. Journal of Telemedicine and Telecare, 11, 146-149.

Kim, J., \& Brienza, D. M. (2006). Development of a Remote Accessibility Assessment System through three-dimensional reconstruction technology. Journal of Rehabilitation Research and Development, 43(2), 257-272.

Kim, J., Brienza, D. M., Lynch, R. D., Cooper, R. A., \& Boninger, M. L. (2008). Effectiveness evaluation of a remote accessibility assessment system for wheelchair users using virtualized reality. Archives of Physical Medicine and Rehabilitation, 89(3), 470-479.

Kinsella, A. (1998). Home Telecare in United States. Journal of Telemedicine and Telecare, 4, 195-200.

Kitt, S. M., \& Clayton, L. (2002). The cost-effectiveness of telehealth in metropolitan hospitals. Journal of Telemedicine and Telecare, 8(Suppl 3), 42-43.

Krupinksi, E., Nypaver, M., Poropatich, R., Ellis, S., Safwat, R., \& Sapci, H. (2002). Clinical applications in telemedicine/telehealth. Telemedicine Journal and e-Health, 8(1).

Lemaire, E. D., Boudrias, Y., \& Greene, G. (2001). Low-bandwidth, internet-based videoconferencing for physical rehabilitation consultations. Journal of Telemedicine and Telecare, 7(2), 82-89. 
Lewis, J. A., Boian, R. F., Burdea, G., \& Deutsch, J. E. (2005). Remote console for virtual rehabilitation. Studies in Health Technology and Informatics(111), 294-300.

Lewis, J. A., Deutsch, J. E., \& Burdea, G. (2006). Usability of the remote console for virtual reality telerehabilitation: formative evaluation. CyberPsychology \& Behavior, 9(2), 142-147.

Liu, L., \& Barlow, I. (2005). The effectiveness and efficiency of using telehealth to provide wheelchair seating assessment and intervention. Paper presented at the IASTED International Conference Alberta, Canada.

Lowitt, M., Kessler, I., Kauffman, C., Hooper, R., Siegel, E., \& Burnett, J. (1998). Teledermatology and in-person examinations: A comparison of patient and physician perceptions and diagnosis agreement. Archives of Dermatology, 134, 471-476.

Mair, F. S., Haycox, A., May, C., \& Williams, T. (2000). A review of telemedicine cost-effectiveness studies. Journal of Telemedicine and Telecare, 6(Suppl 1), 38-40.

Malagodi, M., Schmeler, M. R., Shapcott, N. G., \& Pelleschi, T. (1998, March). The use of telemedicine in assistive technology service delivery: results of a pilot study. Technology Special Interest Section Quarterly, 8.

Mashima, P., Birkmire-Peters, D., Syms, M. J., Holtel, M. R., Burgess, L. P., \& Peters, L. (2003). Telehealth: Voice therapy using telecommunications technology. American Journal of Speech-Language Pathology, 12, 432-439.

Mathewson, C., Adkins, V. K., \& Jones, M. L. (2000). Initial experiences with telerehabilitation and contingency management programs for the prevention and management of pressure ulceration in patients with spinal cord injuries. Journal of Wound, Ostomy and Continence Nursing 27(5), 269-271.

Mathewson, C., Adkins, V. K., Lenyoun, M. A., Schmidt, A. M., \& Jones, M. L. (1999). Using telemedicine in the treatment of pressure ulcers. Ostomy/Wound Management, 45(11), 58-62.

McCue, M., Hodgins, J., LoPresti, E., \& Bargteil, A. (2008). Telerehabilitation in employment/community supports using video-based activity recognition. Telemedicine and e-Health, 14(58).

McCue, M., Lang, F., Bates, J., \& Germek, J. (2003). The effect of cognitive impairments on videoconferencing and telerehabilitation. Technical Report

02-NTSTM104-02: Center of Excellence for Remote and Medically Underserved Areas, July 30.

McCue, M., Pramuka, M., Smathers, B., Germek, J., Muncert, E., \& Lang, F. (2004). A model of "in-vivo" cognitive rehabilitation using multiple telerehabilitation technologies. Technical Report 03-NTSTM111-03: Center of Excellence for Remote and Medically Underserved Areas, July 31.

McKinlay, A., Beattie, W., Arnott, J. L., \& Hine, N. A. (1995). Augmentative and alternative communication: The role of broadband telecommunications. IEEE Transactions on Rehabilitation Engineering, 3(3), 254260.

Ohinamaa, A., \& Hailey, D. (2002). Telemedicine, outcomes, and policy decisions. Disease Management \& Health Outcomes, 10(5), 269-276.
Palsbo, S. E. (2004). Medicaid payment for telerehabilitation. Archives of Physical Medicine and Rehabilitation, 85, 1188-1191.

Pareras, L. G., \& Martin-Rogrigues, J. G. (1996). Neurosurgery and the internet: A critical analysis and a review of available resources. Neurosurgery, 39, 216232.

Perlman, A. L., \& Witthawaskul, W. (2002). Real-Time remote telefluoroscopic assessment of patients with dysphagia. Dysphagia, 17, 162-167.

Philips, V. L., Temkin, A., Vesmarovish, S., \& Burns, R. B. (1998). A feasibility study of video-based home telecare for clients with spinal cord injuries. Journal of Telemedicine and Telecare, 4(4), 219-223.

Philips, V. L., Temkin, A., Vesmarovish, S., Burns, R. B., \& Idleman, L. (1999). Using telehealth interventions to prevent pressure ulcers in newly injured spinal cord injury patients post-discharge. International Journal of Technology Assessment in Health Care, 15(4), 749-755.

Randall, M., Kilpatrick, K. E., Pendergast, J. F., Jones, K. R., \& Vogel, W. B. (1987). Differences in patient characteristics between Veterans Administration and community hospitals Medical Care, 25(11), 1099-1104.

Rehabilitation Research and Training Center on Disability Demographics and Statistics. (2005). 2004 Disability Status Reports. Ithaca, NY: Cornell University.

Ricker, J., Rosenthal, M., Garay, E., DeLuca, J., Germain, A., Abraham-Fuchs, K., et al. (2002). Telerehabilitation needs: A survey of persons with acquired brain injury. The Journal of Head Trauma Rehabilitation, 17(3), 242250.

Roth, A. C., Reid, J. C., Puckett, C. L., \& Concannon, M. J. (1999). Digital images in the diagnosis of wound healing problems. Plastic and Reconstructive Surgery, 103(2), 483-486.

Sabharwal, S., Mezaros, M., \& Duafenbach, L. (2001). Telerehabilitation across the continuum of care for individuals with spinal cord injury. Paper presented at the Proceedings of the State of the Science Conference on Telerehabilitation.

Savard, L., Borstad, A., Tkachuck, J., Lauderdale, D., \& Conroy, B. (2003). Telerehabilitation consultations for clients with neurology diagnoses: Cases from rural Minnesota and American Samoa. NeuroRehabilitation, 18, 93-102.

Schein, R. M., Schmeler, M. R., Brienza, D., Saptono, A., \& Parmanto, B. (2008). Development of a service delivery protocol used for remote wheelchair consultation via telerehabilitation Telemedicine and e-Health, In press.

Schultheis, M. T., \& Rizzo, A. A. (2002). Application of virtual reality technology in rehabilitation. Rehabilitation Psychology, 46(3), 296-311.

Shaw, D. K., Dreyer, N. C., Dreyer, K. A., \& Wittman, P. $P$. (2001). The efficacy of telemedicine in occupational therapy. Journal of Allied Health, 30(1), 39-42.

Sinha, G. (2000). Virtual Healing. Popular Science, 7, 5962.

Soopramanien, A., Pain, H., Stainthrope, A., Menarini, M., \& Ventura, M. (2005). Using telemedicine to provide post-discharge support for patients with spinal cord injuries. Journal of Telemedicine and Telecare, 11 (Suppl 1), 68-70. 
Stausberg, J., \& Person, M. (1999). A process model of diagnostic reasoning in medicine. International Journal of Medical Informatics, 54, 9-23.

Taylor, P. (1998). A survey of research in telemedicine. Journal of Telemedicine and Telecare, 4(2), 63-71.

Theodoros, D. G., Constantinescu, G., \& Russell, T. G. Treating the speech disorder in Parkinson's Disease online. Journal of Telemedicine and Telecare, In press.

Torsney, K. (2003). Advantages and disadvantages of telerehabilitation for persons with neurological disabilities. NeuroRehabilitation, 18, 183-185.

Trepagnier, C. (1999). Virtual environments for the investigation and rehabilitation of cognitive and perceptual impairments. NeuroRehabilitation, 12(1), 63-72.

Trepagnier, C., Noiseux, J., \& Glenshaw, M. (1999, October 13-16). Video communication for vocational support. Paper presented at the Proceedings of the Joint BMES/EMBS Conference Serving Humanity, Advancing Technology, Atlanta.

Vaughn, G. R. (1976). Tele-communicology: Health care delivery system for persons with communicative disorders. ASHA, 18, 13-17.

Vesmarovish, S., Walker, T., Hauber, R. P., Temkin, A., \& Burns, R. B. (1999). Use of telerehabilitation to manage pressure ulcers in persons with spinal cord injuries. Advanced Wound Care, 12, 264.

Wakeford, L. (2002). Telehealth technology for children with special needs. OT Practice, 7(21), 12-16.

Wakeford, L., Whitman, P., White, M. W., \& Schmeler, M. R. (2005). Telerehabilitation position paper. American Journal of Occupational Therapy, 59(6), 656-660.

Whitten, P., Kingsley, C., \& Grigsby, J. (2000). Results of a meta-analysis of cost-benefit research: is this a question worth asking? Journal of Telemedicine and Telecare, 6(Suppl 1), 4-6.

Whitten, P. S., \& Mair, F. (2000). Telemedicine and patient satisfaction: Current status and future directions. Telemedicine Journal and e-Health, 6(4), 417-423.

Winters, J. M. (2002). Telerehabilitation research: emerging opportunities. Annual Review of Biomedical Engineering, 4, 287-320.

Wollinksky, F. D., Coe, R. M., Mosely, R. R., \& Homan, S. M. (1985). Veteran's and no veterans' use of health services. Medical Care, 23(12), 1358-1371.

Zollo, S. A., Kienzle, M. G., Henshaw, Z., Crist, L. G., \& Wakefield, D. S. (1999). Tele-Education in a telemedicine environment: implications for rural health care and academic medical centers Journal of Medical Systems, 23(2), 107-122.
Corresponding address: Mark R. Schmeler, PhD, OTR/L, ATP, University of Pittsburgh

Department of Rehabilitation Science and

Technology

5044 Forbes Tower, Pittsburgh, PA, 15260, USA, Email: schmeler@pitt.edu

This document is a draft intended for use by

participants in the Rehabilitation Engineering

Research Center on Telerehabilitation's State of

the Science Conference to be held November

17-20, 2008. The document may not be distributed

and should be discarded after the conference.

A final draft will be developed in response to the

discussions taking place during the conference.

The paper will be published in final form in the

April 2009 issue of the International Journal of

Telerehabilitation. We look forward to your active

participation in the conference and welcome any

comments you have related to the information

presented here. 